\title{
Capture and Release of Target Cells Using a Surface That Immobilizes an Antibody via Desthiobiotin-Avidin Interaction
}

\author{
Tsuyoshi Kimura, ${ }^{*}$ Naoko Nakamura, Nao Sasaki, Yoshihide Hashimoto, \\ Shimon Sakaguchi, ${ }^{1}$ Shunsaku Kimura, ${ }^{2}$ and Akio Kishida \\ Institute of Biomaterials and Bioengineering, Tokyo Medical and Dental University, \\ 2-3-10 Kanda-Surugadai, Chiyoda-ku, Tokyo 101-0062, Japan \\ ${ }^{1}$ Immunology Frontier Research Center, Osaka University, Yamadaoka, Suita, Osaka 565-0871, Japan \\ ${ }^{2}$ Graduate School of Engineering, Kyoto University, Kyoto Daigaku-Katsura, Nishikyo-ku, Kyoto 615-8530, Japan
}

(Received March 29, 2016; accepted September 6, 2016)

Keywords: selective cell capture, cell release, antibody immobilization, surface modification

We designed and synthesized a polyethylene film that immobilizes antibodies via desthiobiotinavidin interaction by grafting poly(acrylic acid) (PAAc) and biotin to its surface. The desthiobiotinmodified antibodies (anti-mouse CD45 and CD25 antibodies) were immobilized on the film through desthiobiotin-avidin interaction. Mouse bone marrow (CD45-positive) and HL60 (CD45negative) cells were seeded on the films with and without immobilizing the anti-mCD45 antibody, and the selective adhesion of cells was observed. Also, mouse spleen cells (CD25-positive) were selectively captured on the anti-mCD25-antibody-immobilized film. These captured cells were completely released by the addition of biotin-conjugated water-soluble polymers, indicating that the dissociation of desthiobiotin-avidin complexes occurred owing to the different association constants of desthiobiotin and biotin to avidin. From these results, it was suggested that target cells could be selectively captured and collected using a film having a surface that immobilizes an antibody via desthiobiotin-avidin interaction.

\section{Introduction}

Cell separation methods are widely used in cell biology and immunology. The isolated cells, which possess therapeutic functions, are applied to cell therapy, immune therapy, and regenerative medicine. Generally, the phenotypic or functional features of cells, such as size, morphology, density, surface charge, antigen, cytoplasmic composition, and other properties, are used as a basis for cell separation. Cell separation methods are mainly classified into physical separation, fluorescence-activated cell sorting (FACS), and magnetic-activated cell sorting (MACS). ${ }^{(1-3)}$ Physical separation based on the difference in the specific gravity of cells is a simple and lowcost method. For FACS, cell components are labeled with a fluorescent dye and individual cells are measured fluorescently through a liquid stream and then sorted. For MACS, magneticbeads-labeled cells are trapped with a column in a magnetic field and collected. Also, recently, microfluidic $^{(4,5)}$ and surface modification ${ }^{(6)}$ techniques have been studied for cell separation. These

"Corresponding author: e-mail: kimurat.mbme@tmd.ac.jp 
methods have advantages and disadvantages and are employed in different ways for different purposes.

Regulatory T (Treg) cells expressing CD4, CD25 and the transcription factor forkhead box P3 play important roles in maintaining immunological self-tolerance and in suppressing excessive immune responses. ${ }^{(7)}$ Recent research has revealed that Treg cells suppress the activation of tumorantigen-specific $\mathrm{T}$ cells. To evoke antitumor immunity, they can be one of the target cells, and reducing the number of Treg cells from cancer patients is an important approach to treatment. ${ }^{(8,9)}$ Thus, the number of Treg cells should be selectively reduced from a cancer patient's body, e.g., peripheral blood, while retaining functional cells. In many cases of the cell separation described above, cells are harvested from the body and the cell suspension is used in vivo with nontarget cells being discarded. Therefore, it is ideal to develop a device, e.g., a column and a filter, that can capture Tregs selectively through extracorporeal circulation. Also, the captured Treg cells of the host patient have the potential to promote transplant immune tolerance. ${ }^{(10)}$ The collection of captured cells is also one of the important techniques for such treatments.

Given this background, our final goal is to develop a device that can capture and collect target cells from the blood, selectively and effectively, for its use in cell therapy. In this study, we developed a surface on which target cells are captured and released selectively. An antibody was modified with desthiobiotin and immobilized on a surface by binding to avidin. Using this surface, the selective capture and release (collection) of target cells were investigated (Fig. 1).

\section{Materials and Methods}

\subsection{Materials}

A polyethylene (PE) film (thickness: $50 \mu \mathrm{m}$ ) was purchased from Acrysunday Co., Ltd. (Tokyo, Japan). Anti-mouse CD45 (mCD45) antibody was purchased from BD Biosciences, Japan (Tokyo, Japan). Anti-mouse CD25 (mCD25) antibody was obtained from a rat. EZ-Link Amine$\mathrm{PEG}_{2}$-biotin, EZ-Link NHS-desthiobiotin, avidin, a biotin quantitation kit, phosphate-buffered saline (PBS), and RPMI1640 cell culture medium were purchased from Thermo Fisher Scientific K.K. (Yokohama, Japan). Fetal bovine serum (FBS) was purchased from Life Technologies Japan (Tokyo, Japan). 1-Ethyl-3-(3-dimethylaminopropyl) carbodiimide hydrochloride (EDC), biotin $\mathrm{N}$-hydroxysulfosuccinimide ester (Biotin-Sulfo-OSu), 2-morpholinoethanesulfonic acid monohydrate (MES), and CytoRed ${ }^{\circledR}$ were purchased from Dojindo Laboratories (Kumamoto,

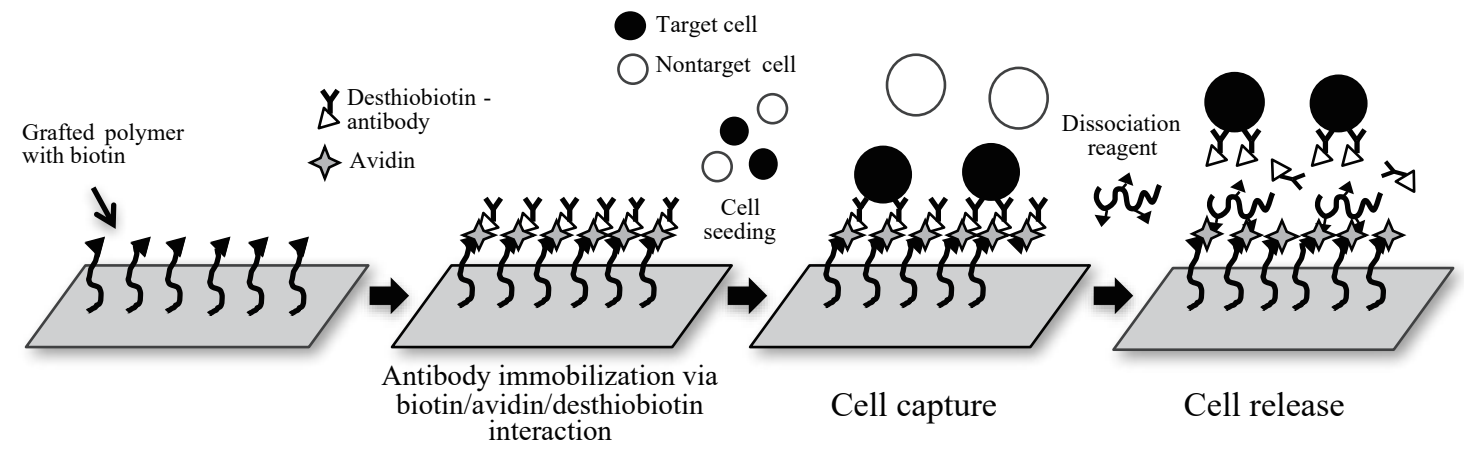

Fig. 1. Selective capture and release of target cells on a desthiobiotin-antibody-immobilized surface. 
Japan). Acrylic acid was purchased from Wako Pure Chemical Industries, Ltd. (Osaka, Japan) and used after distillation. Poly(acrylic acid) (PAAc, Mw: 250,000) and sodium carboxymethyl dextran (CM-dex, Mw: 1,000,000) were purchased from Sigma-Aldrich Japan Inc. (Tokyo, Japan).

\subsection{Preparation of PAAc-grafted film (PAAc-g-film)}

Films were washed by soxhlet extraction for $24 \mathrm{~h}$. The films were treated by corona discharge treatment at $15 \mathrm{kV}$ for $1 \mathrm{~min} .{ }^{(11,12)}$ Five to ten films $\left(10 \times 50 \mathrm{~mm}^{2}\right)$ were set in glass tubes and $5 \mathrm{ml}$ of $5 \mathrm{w} / \mathrm{v} \%$ acrylic acid solution was added. After degassing, the tubes were closed and incubated at $60{ }^{\circ} \mathrm{C}$ for $30 \mathrm{~min}$ for graft polymerization. The PAAc-g-film were washed with deionized water and dried in ambient air (Fig. 2).

The surface wettability of the PAAc-g-film was measured using FTA1000 (First Ten Angstroms, Portsmouth, USA). Five to ten independent measurements at different sites of one sample were averaged. Deionized water was used for the measurement. These experiments were carried out five times. Values represent mean \pm standard error.

\subsection{Preparation of the antibody-immobilized PAAc film}

Anti-mCD45 and anti-mCD25 antibodies were diluted in PBS at the concentration of $0.5 \mathrm{mg} /$ $\mathrm{ml}$. Eight hundred microliter of the antibody solution was mixed with $50 \mu 1$ of NHS-desthiobiotin $(8 \mathrm{mM})$ or biotin-sulfo-Osu $(8 \mathrm{mM})$ and then incubated at room temperature for $2 \mathrm{~h}$ in order to conjugate the antibody with desthiobiotin or biotin. The modified antibody was purified by gel permeation chromatography (PD-10 column: GE Healthcare, Ltd., UK). PAAc-g-films of $9 \mathrm{~mm}$ diameter were set in a 48-well cell culture plate and $200 \mu 1$ of 0.1 M MES buffer ( $\mathrm{pH} 5.5$ ) was added. Two hundred microliter of amine-PEG ${ }_{2}$-biotin $(1 \mathrm{mM})$ and $200 \mu 1$ of EDC $(10 \mathrm{mg} / \mathrm{ml})$ were added and incubated at room temperature for $2 \mathrm{~h}$. The biotin-immobilized film was washed
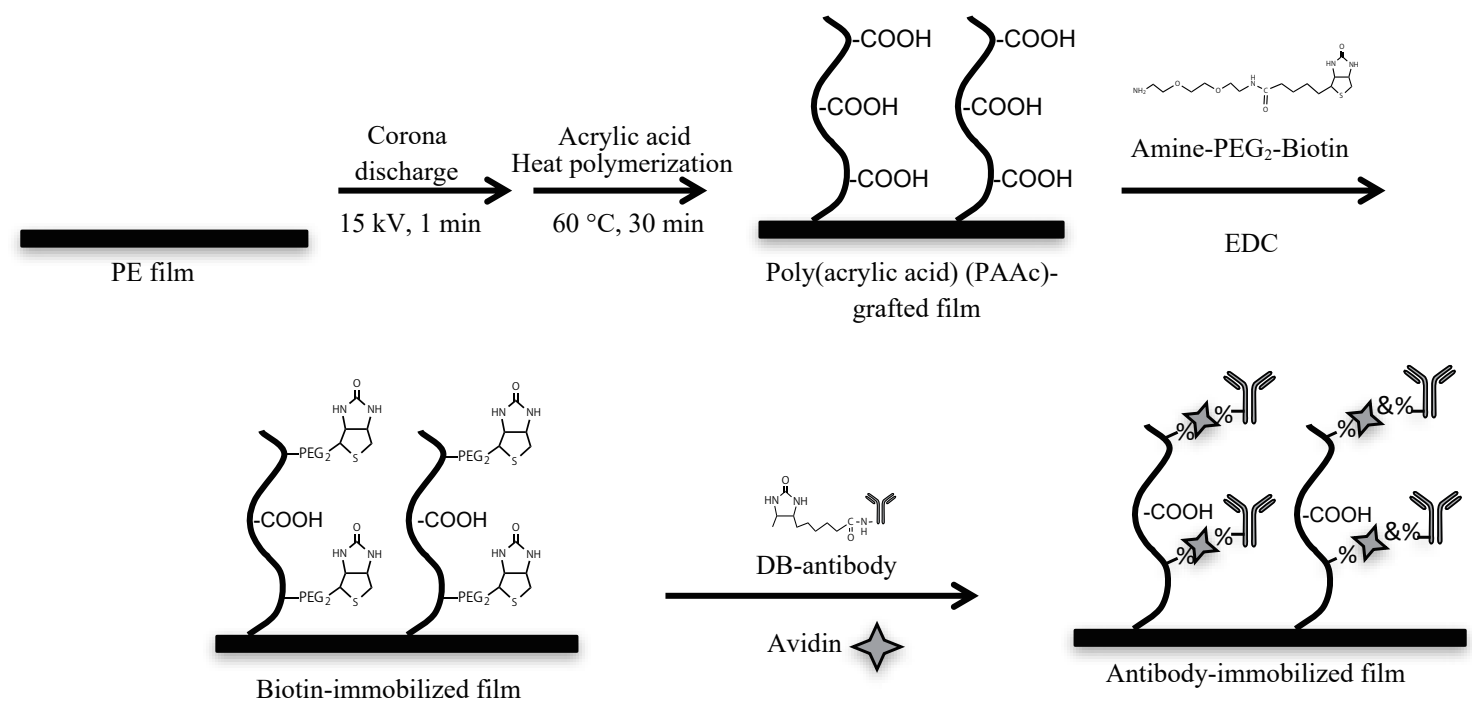

Fig. 2. Preparation of a desthiobiotin-antibody-immobilized film. B: biotin, DB: desthiobiotin. 
with PBS three times. One hundred microliter of the avidin solution $(2 \mathrm{mg} / \mathrm{ml}), 100 \mu \mathrm{l}$ of the desthiobiotin-antibody or biotin-antibody solution, and $100 \mu 1$ of PBS were applied to the biotinimmobilized film in order to immobilize the antibody on the film through desthiobiotin-avidin and biotin-avidin interactions. After incubation for $12 \mathrm{~h}$ at $4{ }^{\circ} \mathrm{C}$, the anti-mCD45- or anti-mCD25antibodies-immobilized films were used after washing with PBS three times.

To investigate the immobilization of the antibody on the film, the film was stained using an immunostaining kit (TaKaRa POD Conjugate Anti-rat, for mouse tissue) following the manufacturer's instructions.

\subsection{Preparation of dissociation agent}

Forty milliliter of PAAc solution $(25 \mathrm{mg} / \mathrm{ml}$ in $0.1 \mathrm{M}$ MES) was mixed with $10 \mathrm{ml}$ of EDC $(133 \mathrm{mg} / \mathrm{ml}$ in $0.1 \mathrm{M} \mathrm{MES})$ and $8.35 \mathrm{ml}$ of amine-PEG ${ }_{2}$-biotin $(10.2 \mathrm{mg} / \mathrm{ml}$ in DMSO). Forty milliliter of CM-dex solution ( $25 \mathrm{mg} / \mathrm{ml}$ in $0.1 \mathrm{M} \mathrm{MES})$ was mixed with $10 \mathrm{ml}$ of EDC $(42.2 \mathrm{mg} /$ $\mathrm{ml}$ in $0.1 \mathrm{M} \mathrm{MES})$ and $8.35 \mathrm{ml}$ of amine-PEG - -biotin $(3.2 \mathrm{mg} / \mathrm{ml}$ in DMSO). They were reacted at room temperature for $3 \mathrm{~h}$. The biotin-modified PAAc or CM-dex was purified by dialysis (cutoff Mw: 50,000) for 2 days and freeze-dried. The biotin concentration was measured using a biotin quantitation kit (HABA method), following the manufacturer's instructions.

\subsection{Specific cell capture}

EGFP mice (C57BL/6-Tg, male, 10-20 g) were purchased from Sankyo Lab Service Corp. Bone marrow (BM) cells were harvested from the femur bone by the bone marrow flashing method. HL60 cells were stained with CytoRed ${ }^{\circledR}$ following the manufacturer's instructions. A mixture of BM and HL60 cells in RPMI1640 medium was seeded on the antibody-immobilized films at the cell densities of $7 \times 10^{4}$ and $7 \times 10^{5}$ cells $/ \mathrm{cm}^{2}$, respectively. The cells were incubated at $4{ }^{\circ} \mathrm{C}$ for $1 \mathrm{~h}$ to enhance the antibody-antigen interaction by excluding cell-substrate adhesion mediated by cell adhesion molecules. Nonadhered cells were removed by washing with PBS three times. The adhered (captured) cells were observed under a fluorescence microscope and the number of cells was counted. These experiments were carried out three times. Values represent mean \pm standard error. Student's t-test was used for statistical analyses.

\subsection{Cell release using dissociation reagent}

EGFP-BM cells were seeded on the anti-mCD45 antibody-immobilized film at the density of 3.5 $\times 10^{5}$ cells $/ \mathrm{cm}^{2}$. Also, EGFP-spleen cells were seeded on the anti-mCD25 antibody-immobilized film at the density of $7 \times 10^{5}$ cells $/ \mathrm{cm}^{2}$. After $1 \mathrm{~h}$ of incubation at $4{ }^{\circ} \mathrm{C}$, the nonadhered cells were washed out. The adhered cells were observed under a fluorescence microscope and the number of cells was counted. To release the adhered cells, the dissociation reagents biotin-PAAc or biotin$\mathrm{CM}$-dex were added at the biotin concentration of $10 \mu \mathrm{M}$. After 10 min of incubation at room temperature, the released cells were removed by washing with PBS. The adhered cells were observed under a fluorescence microscope and the number of cells was counted. These experiments were carried out three times. Values represent mean \pm standard error. Student's t-test was used for statistical analyses. 


\section{Results}

\subsection{Preparation of antibody-immobilized film}

The surface of PE film was oxidized by corona discharge and, subsequently, acrylic acid was graft-polymerized onto the film surface. The PAAc-g-film was stained with methylene blue, which electrostatically interacts with the carboxyl moiety of PAAc. Nontreated and corona-discharged films were not stained. On the other hand, the PAAc-g-film was stained strongly (data not shown). The contact angle of the PAAc-g-film for water was about $19 \pm 2^{\circ}$, indicating the PAAc grafting to the film surface (Fig. 3).

The anti-mCD45 or anti-mCD25 antibody was conjugated with desthiobiotin or biotin and then immobilized on the biotin-modified PAAc-g-film through desthiobiotin-avidin and biotin-avidin interactions. By immunostaining, the immobilization of the antibody on the film was confirmed. The antibody-immobilized films through desthiobiotin-avidin and biotin-avidin interactions became uniformly brown (Fig. 4).

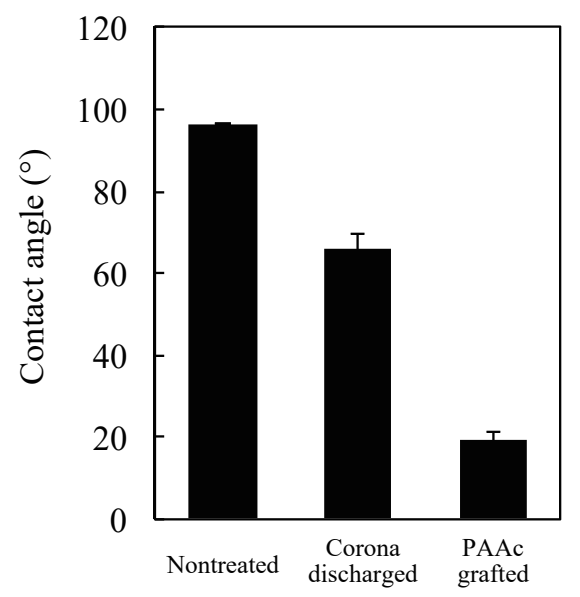

Fig. 3. Contact angle of nontreated PE film, corona-discharged PE film, and PAAc-g-film. Values represent mean \pm standard error. $n=3$.

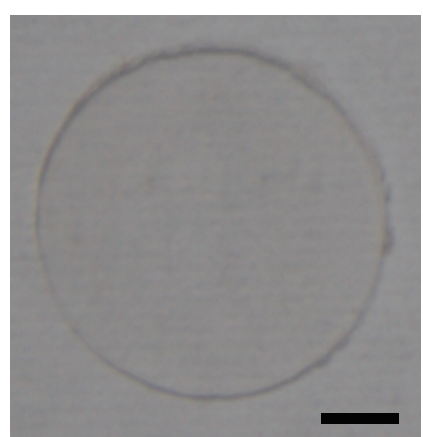

(a)

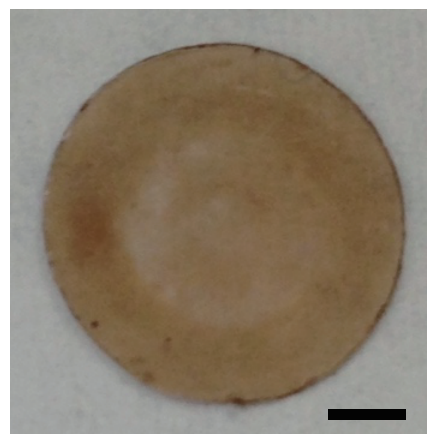

(b)

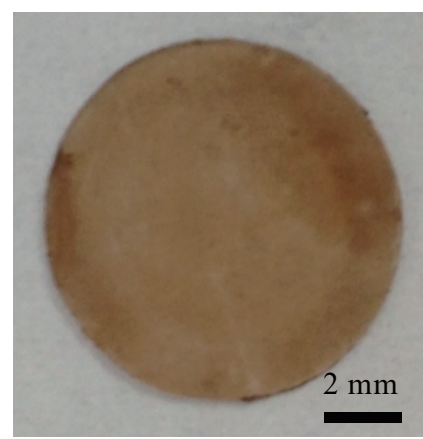

(c)

Fig. 4. (Color online) Immunostaining of antibody-immobilized PE films. (a) Nontreated PE film, (b) biotinmCD45 antibody, and (c) desthiobiotin-mCD45 antibody. 


\subsection{Specific cell capture}

HL60 cells are CD45-negative cells. BM cells include about 88\% of CD45-positive cells. HL60 cells were stained with CytoRed $^{\circledR}$, which is a red fluorescent dye for labeling living cells. A mixture of HL60 and EGFP-BM cells (green cells) was seeded on the anti-mCD45-antibodyimmobilized film. After $1 \mathrm{~h}$ of incubation at $4{ }^{\circ} \mathrm{C}$, the nonadhered cells were washed out using PBS and the adhered cells were observed under a fluorescence microscope. Figure 5 shows the fluorescence microscopy image and the number of adhered cells. Using the corona-discharged film, many red HL60 cells and green BM cells were observed. The percentages of adhered cells were about $55 \pm 2 \%$ and $36 \pm 14 \%$ for HL60 and BM cells, respectively. For the PAAc-g-film, a few adhered HL60 (about $0.5 \pm 0.2 \%)$ and BM $(4.6 \pm 2.0 \%)$ cells were observed. On the other hand, when the anti-mCD45-antibody-immobilized film was used, many green BM (CD45-positive) cells were observed uniformly and the percentage of adhered cells was about $57 \pm 13 \%$, indicating the specific and selective capture of target cells through antibody-antigen interaction on this antibodyimmobilized film.

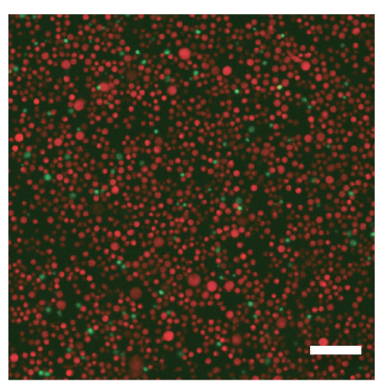

(a)

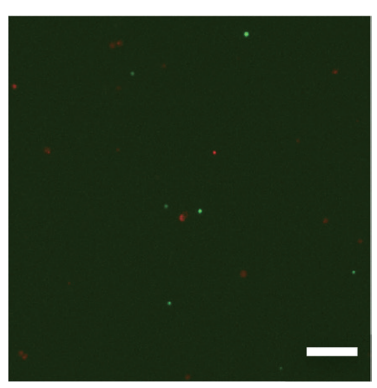

(b)

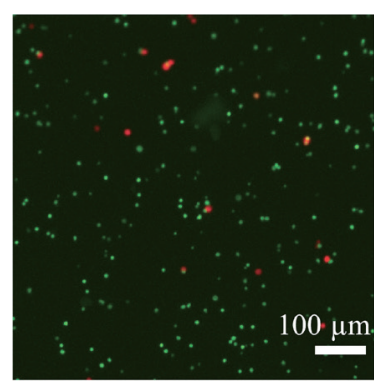

(c)

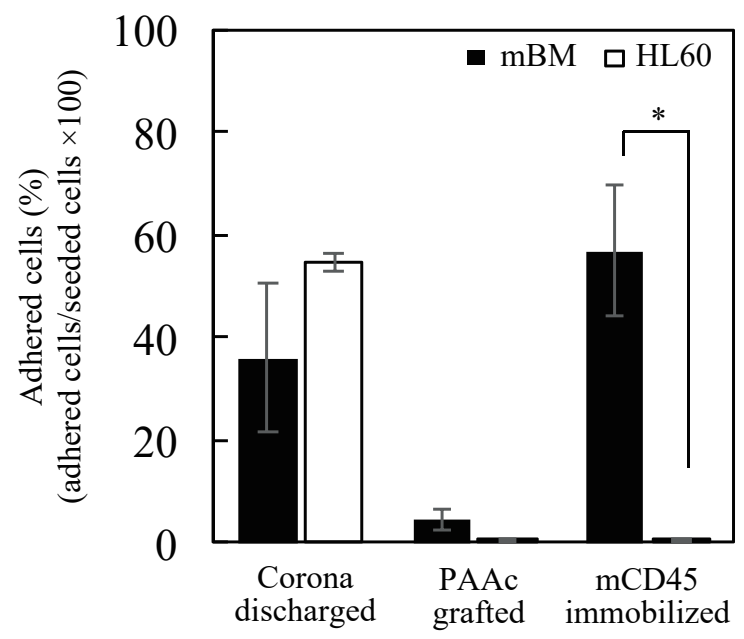

(d)

Fig. 5. (Color online) Selective capture of target cells on the antibody-immobilized PE film. Fluorescent images of the adhered HL60 cells (red) and EGFP-BM cells (green). (a) Corona-discharged film, (b) PAAc-g-film, and (c) mCD45-immobilized film. (d) Percentages of adhered cells. Values represent mean \pm standard error. $n=3$. ${ }^{*} P<0.05$. 


\subsection{Release of captured cells}

EGFP-BM cells were seeded on the mCD45-antibody-immobilized film with desthiobiotinmCD45 antibody and biotin-mCD45 antibody. After washing with PBS, the biotin-PAAc and biotin-BSA solutions, which are dissociation reagents, at the biotin concentrations of 10 and 17 $\mu \mathrm{M}$ were added, respectively. Figure 6 shows the fluorescence microscopy image and number of adhered cells before and after the addition of dissociation reagents. In both cases of desthiobiotinmCD45 and biotin-mCD45, similar amounts of BM cells adhered [Figs. 6(a), 6(b), and 6(e)]. By using the biotin-BSA, the adhered cells were not released. By using biotin-PAAc for the biotinmCD45-antibody-immobilized film, about $33 \pm 6 \%$ of the seeded cells still adhered. On the other hand, for the desthiobiotin-mCD45 antibody-immobilized film, the adhered cells were completely released [Figs. 6(d) and 6(e)]. When BM cells were stained with propidium iodide (PI), which is a dead cell marker, after adding dissociation reagent, there was no big difference in the percentage of PI-positive cells between the nontreated cells and the treated cells with dissociation reagent (data not shown).

By using the mCD25-antibody-immobilized film, the spleen, in which about $45 \%$ of CD25positive cells are included, was captured effectively (Fig. 7). By adding biotin-CM-dex at the biotin concentration of $10 \mu \mathrm{M}$, the cells were effectively released.
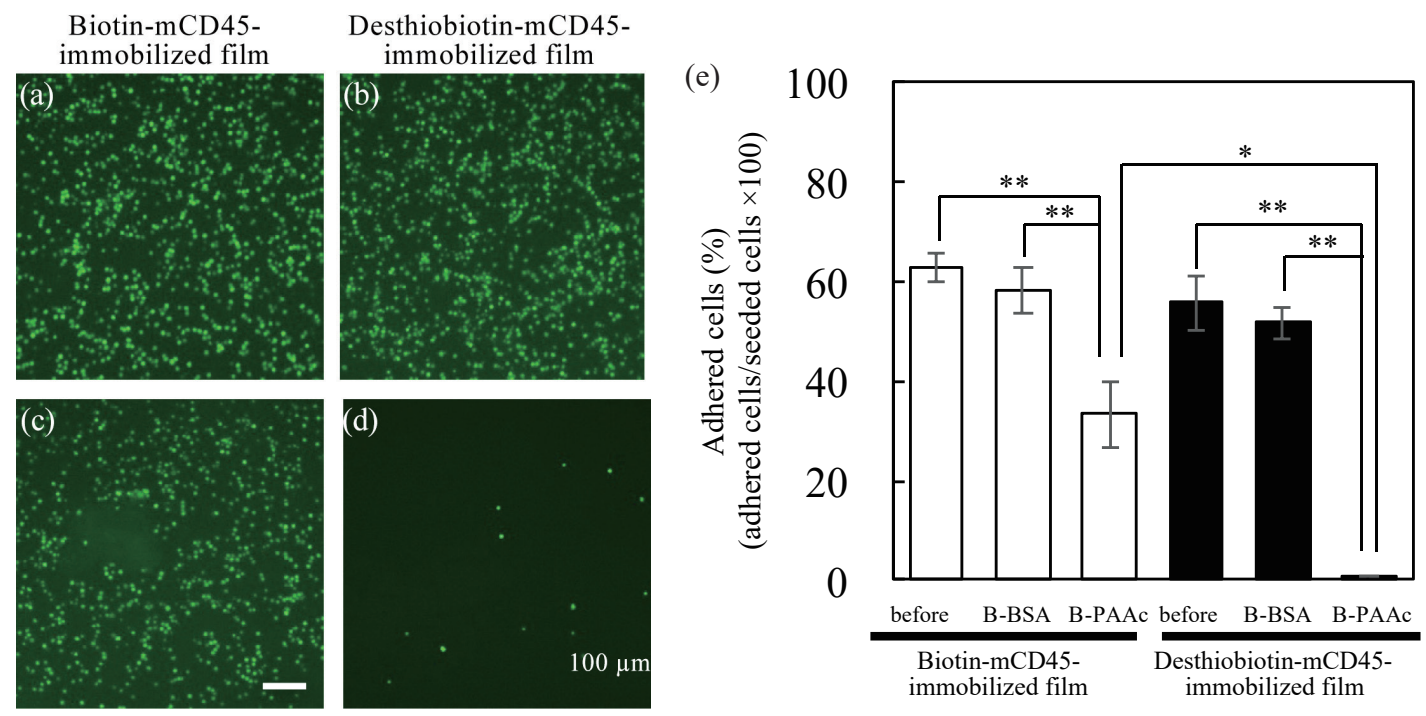

Fig. 6. (Color online) Release of captured cells on biotin-mCD45- and desthiobiotin-mCD45-immobilized PE films by the addition of dissociation reagents. Fluorescent images of adhered EGFP-BM cells. (a), (b) Before addition of biotin-PAAc, (c), (d) after addition of biotin-PAAc, (a), (c) biotin-mCD45-immobilized film, (b), (d) desthiobiotin-mCD45-immobilized film. (e) Percentages of adhered cells before and after the addition of biotinBSA (B-BSA, $17 \mu \mathrm{M}$ ) and biotin-PAAc (B-PAAc, $10 \mu \mathrm{M}$ ). Values represent mean \pm standard error. $n=3$. ${ }^{*} P<0.02$, ${ }^{* *} P<0.01$. 


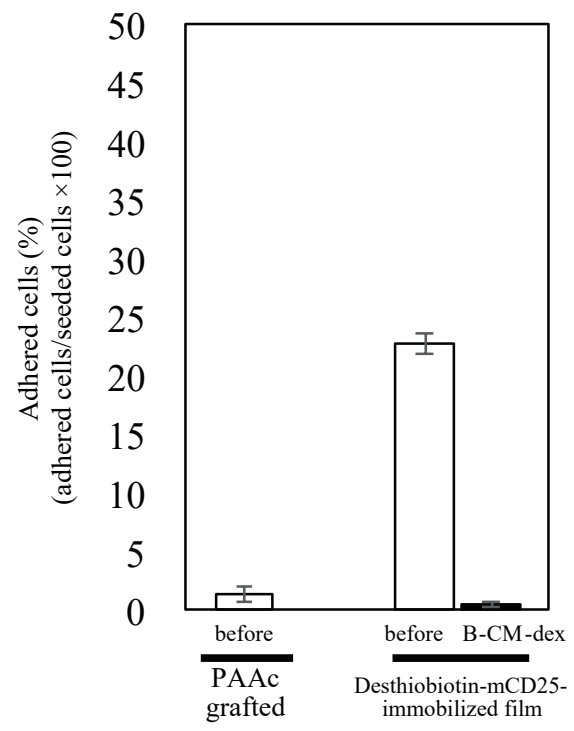

Fig. 7. Capture and release of spleen cells on desthiobiotin-mCD25-immobilized PE film before and after the addition of biotin-CM-dex (B-CM-dex). Values represent mean \pm standard error. $n=3$.

\section{Discussion}

Our final aim is to develop a device that can capture and collect target cells from the blood, selectively and effectively, for its use in cell therapy. In this study, we designed and synthesized a surface that immobilizes an antibody through desthiobiotin-avidin interaction in order to capture and release target cells effectively and selectively (Fig. 1). Acrylic acid was graft-polymerized onto the surface of the PE film because it could inhibit the adhesion of nontarget cells. ${ }^{(12)}$ When cells were seeded on the PAAc-g-film, no cellular adhesion was observed, indicating the inhibition of the nonspecific adhesion of cells (Figs. 5 and 7). For immobilizing the antibody on the surface, the antibody was conjugated with desthiobiotin. Desthiobiotin as well as biotin can bind to avidin specifically and is applicable for labeling and immobilizing molecules. ${ }^{(13,14)}$ The binding affinity of desthiobiotin to avidin is lower than that of biotin (Ka: desthiobiotin: $5 \times 10^{13} \mathrm{M}^{-1}$, biotin: $\sim 1 \times$ $10^{15} \mathrm{M}^{-1}$ ), and desthiobiotin-avidin complexes are dissociated by the addition of biotin owing to the exchange reaction between desthiobiotin and biotin. ${ }^{(13-15)}$ In this study, the desthiobiotin-antibody was immobilized on the surface by binding to avidin in order to release the captured cells. The immobilized antibody could be released by the addition of biotin. By using the mCD45-antibodyimmobilized film via desthiobiotin-avidin interaction, cells, including target BM (CD45-positive) and nontarget HL60 (CD45-negative) cells, were seeded and the BM cells were captured uniformly and selectively (Fig. 5). These results indicate that the cells interacted with the immobilized mCD45 antibody keeping its activity. Subsequently, the captured cells were effectively dissociated by the addition of biotin-PAAc, suggesting that the complexes of avidin and desthiobiotin-antibody, which reacted with cellular antigen, underwent a dissociation (replacement) reaction with the biotin of PAAc (Fig. 6). On the other hand, no effective release of cells was observed using biotinBSA. Although the mechanism was unclear, there may be an optimal condition for biotin-BSA. It is necessary to further investigate the detailed mechanism. CD25-positive spleen cells were also 
captured on the desthiobiotin-mCD25 antibody-immobilized film and then released by the addition of biotin-CM-dex (Fig. 7). These results strongly suggest that the capture and release of target cells were achieved using the antibody-immobilized film via desthiobiotin-avidin interaction. In this study, we used two types of dissociation reagents, biotin-PAAc and biotin-CM-dex. First, the biotin-PAAc was used in terms of compatibility to the PAAc graft chain of film. To operate the dissociation reagent easily, the biotin-CM-dex with lower viscosity than biotin-PAAc was also used because there was no big difference in the dissociation ability of either of them (data not shown). It is also assumed that the cell-releasing effect depends on many factors, such as the biotin modification ratio of the dissociation reagent, the molecular weight of the polymer, the cell type, the antibody, and the amount of immobilized antibody. The percentages of CD45-positive cells in BM cells and CD25-positive cells in spleen cells were about 88 and 45\%, respectively. About $57 \pm 13 \%$ of the seeded BM cells and $22.8 \pm 1.0 \%$ of the seeded spleen cells were captured. To improve the efficacy of capturing and releasing target cells, further studies are needed. Also, the features of the collected cells, such as viability and functions, will be investigated in the future.

\section{Conclusions}

We successfully developed a surface that immobilizes an antibody via desthiobiotin-avidin interaction for the capture and release of target cells. For the antibody-immobilized surface, target cells were captured through antibody-antigen reaction, effectively and selectively, and then released using biotin-PAAc and biotin-CM-dex due to an exchange reaction of desthiobiotin and biotin. This surface would be useful for use as a cell-capturing and cell-releasing device.

\section{Acknowledgements}

This work was partly supported by the Strategic Promotion of Innovative Research and Development Division of the Japan Science and Technology Agency.

\section{References}

1 R. Cramer, P. Dri, G. Zabucchi, and P. Patriarca: J. Leukoc. Biol. 52 (1992) 331.

2 E. A. O’Donnell, D. N. Ernest, and R. Hingorani: Immune Network 13 (2013) 43.

3 S. Miltenyi, W. Muller, W. Weichel, and A. Radbruch: Cytometry 11 (1990) 231.

4 S. K. Sia and G. M. Whitesides: Electrophoresis 24 (2003) 3563.

5 C. Wyatt Shilds IV, C. D. Reyes, and G. P. Lopez: Lab. Chip 15 (2015) 1230.

6 J. Kobayashi, M. Hayashi, T. Ohno, M. Nishi, Y. Arisaka, Y. Matsubara, H. Kakidachi, Y. Akiyama, M. Yamato, A. Horii, and T. Okano: J. Biomed. Mater. Res. Part A 102 (2014) 3883.

7 S. Sakaguchi, T. Yamaguchi, T. Nomura, and M. Ono: Cell 133 (2008) 775.

8 H. Nishikawa and S. Sakaguchi: Int. J. Cancer 127 (2010) 759.

9 H. Nishikawa and S. Sakaguchi: Curr. Opin. Immun. 27 (2014) 1.

10 S. Jiang, J. Tsang, and P. Tam: Int. Immunopharm. 10 (2010) 1486.

11 H. Iwata, A. Kishida, M. Suzuki, Y. Hata, and Y. Ikada: J. Polym. Sci. Part A 26 (1988) 3309.

12 A. Kishida, H. Iwata, Y. Tamada, and Y. Ikada: Biomaterials 12 (1991) 786.

13 J. D. Hirsch, L. Eslamizar, B. J. Filanoski, N. Malekzadeh, R. P. Haugland, J. M. Beechem, and R. P. Haugland: Anal. Biochem. 308 (2002) 343.

14 H. C. Yoon, M. Y. Hong, and H. S. Kim: Langmuir 17 (2001) 1234.

15 H. Inoue, K. Sato, and J. Anzai: Biomacromolecules 6 (2005) 27. 